\title{
Prognostic and clinicopathological value of CD90 expression in cancer patients: a systematic review and meta-analysis
}

\author{
Hyun Min Koh ${ }^{1}$, Hyun Ju Lee ${ }^{2,3}$, Dong Chul Kim ${ }^{4,5,6}$ \\ ${ }^{1}$ Department of Pathology, Gyeongsang National University Changwon Hospital, Changwon, Korea; ${ }^{2}$ Department of Pathology, Soonchunhyang \\ University College of Medicine, Cheonan, Korea; ${ }^{3}$ Department of Pathology, Soonchunhyang University Cheonan Hospital, Cheonan, Korea; \\ ${ }^{4}$ Department of Pathology, Gyeongsang National University School of Medicine, Jinju, Korea; ${ }^{5}$ Department of Pathology, Gyeongsang National \\ University Hospital, Jinju, Korea; ${ }^{6}$ Gyeongsang Institute of Health Science, Jinju, Korea \\ Contributions: (I) Conception and design: All authors; (II) Administrative support: HM Koh, DC Kim; (III) Provision of study materials or patients: \\ HM Koh, DC Kim; (IV) Collection and assembly of data: HM Koh, HJ Lee; (V) Data analysis and interpretation: HM Koh, HJ Lee; (VI) Manuscript \\ writing: All authors; (VII) Final approval of manuscript: All authors. \\ Correspondence to: Hyun Ju Lee, MD, PhD. Department of Pathology, Soonchunhyang University Cheonan Hospital, Soonchunhyang University \\ College of Medicine, 31 Soonchunhyang 6 gil, Cheonan 31151, Korea. Email: drlhjmd@daum.net; Dong Chul Kim, MD, PhD. Department of \\ Pathology, Gyeongsang National University School of Medicine, 79 Gangnam-ro, Jinju 52727, Korea. Email: kdcjes@gmail.com.
}

\begin{abstract}
Background: Recent studies have shown that CD90 has an important role in cancer development. Moreover, CD90 is reportedly associated with cancer progression, metastasis, and poor prognosis. Thus, we performed this meta-analysis to investigate the prognostic and clinicopathological value of CD90 expression in patients with cancer.

Methods: Eligible studies were collected by searching PubMed, Embase, and the Cochrane library. The pooled results were analyzed to reveal the association between CD90 expression and survival as well as the clinicopathological characteristics of cancer patients.

Results: CD90 overexpression was associated with poor survival in cancer patients [for overall survival, hazard ratio (HR): 2.56, 95\% confidence interval (CI): 1.42-4.62, $\mathrm{P}=0.002$; for disease-free survival, HR: 1.88, 95\% CI: $1.08-3.27, \mathrm{P}=0.025$ ] and was also significantly correlated with a larger tumor size [odds ratio (OR): 1.97, 95\% CI: 1.01-3.85, P=0.048), higher tumor grade (OR: 2.72, 95\% CI: 1.33-5.54, P=0.006), lymph node metastasis (OR: 3.66, 95\% CI: 1.14-11.78, $\mathrm{P}=0.029)$, and higher tumor-node-metastasis stage (OR: 4.79, 95\% CI: 2.28-10.04, P<0.001).
\end{abstract}

Conclusions: CD90 overexpression could predict poor prognosis and may hence be a potential prognostic biomarker for cancer patients.

Keywords: Cancer; CD90; meta-analysis; prognosis

Submitted Feb 09, 2021. Accepted for publication May 14, 2021.

doi: $10.21037 /$ tcr-21-266

View this article at: https://dx.doi.org/10.21037/tcr-21-266

\section{Introduction}

Cancer significantly contributes to overall morbidity and mortality (1). Therefore, it is very important to predict cancer progression and to classify and treat patients accordingly. Recently, there have been numerous attempts to utilize biomarkers in early detection and treatment.

CD90 is a glycophosphatidylinositol-anchored cell surface protein (2). It regulates cell adhesion, migration and proliferation, apoptosis, and cellular communication and is involved in T cell activation, wound healing, and fibrosis $(2,3)$. CD90 is expressed in various cells such as mesenchymal and hematopoietic stem cells, neurons, endothelial cells, and fibroblasts $(2,3)$. Recent studies have shown that CD90 also has an important role in the development of various cancers, including liver, gastric, and 
esophageal cancers (4). Moreover, it has been shown that CD90 is related to cancer progression, metastasis, and poor patient survival (4-9).

Nevertheless, little is known about the relationship between CD90 expression and the prognosis of cancer patients. Thus, we systematically investigated the prognostic and clinicopathological value of CD90 expression in cancer.

We present the following article in accordance with the PRISMA reporting checklist (available at https://dx.doi. org/10.21037/tcr-21-266).

\section{Methods}

\section{Literature search}

We performed a literature search in PubMed, Embase, and the Cochrane library until June 2020 using the following keywords: "CD90" and "cancer or carcinoma or malignancy" and "prognosis or survival or outcome". We also performed an additional manual search.

\section{Inclusion and exclusion criteria}

The inclusion criteria were as follows: (I) CD90 expression identified in human cancer tissue using immunohistochemistry and (II) the hazard ratio (HR) with 95\% confidence interval (CI) between CD90 expression and clinical outcome should be available. The following articles were excluded: (I) duplicate articles, (II) a study of pediatric patients, and (III) reviews, conference abstracts, and nonEnglish articles.

\section{Data extraction and quality assessment}

Each of the two authors extracted basic data such as the first author, year of publication, country, cancer type, sample size, sex, mean or median age of patients, study period, follow-up period, clinical outcome, and cutoff value of CD90 expression. The Newcastle-Ottawa Scale was used to assess the quality of the included studies. In case of a disagreement, the authors reached an agreement through discussion.

\section{Statistical analysis}

Data were analyzed using StataSE12 (Stata, College Station, TX, USA), and statistical significance was determined only if the $\mathrm{P}$ value was less than $0.05 \mathrm{I}^{2}$ statistics were used to check for heterogeneity between the included studies. A random effects model was applied if the $\mathrm{I}^{2}$ statistic was $>50 \%$ or the $\mathrm{P}$ value was $<0.1$; otherwise, a fixed model was applied. The pooled HR and odds ratio (OR) were calculated to evaluate the prognostic and clinicopathological value of CD90 expression. A funnel plot (using Egger's test) and a filled funnel plot were constructed to check for publication bias, and sensitivity analysis was performed to prove the reliability of the pooled HR.

\section{Results}

\section{Study selection and basic data}

We included six eligible studies through the process presented in Figure 1. The basic data of these studies are given in Table 1. The reports consisted of hepatocellular carcinoma $(\mathrm{n}=2)$, intrahepatic cholangiocarcinoma $(n=1)$, gallbladder cancer $(n=1)$, breast cancer $(n=1)$, and chondrosarcoma $(n=1)$. Four studies originated from China, and the other two studies were published in Brazil and Japan, respectively. The minimum, maximum, and total number of samples was 50, 278, and 676, respectively. All of the studies were verified to be of good quality with eight scores.

\section{The association between CD90 expression and overall survival (OS)}

Of the included studies, five reported the relationship between CD90 expression and OS. Zhang et al. (8) reported the relationship between CD90 expression and OS in squamous cell/adenosquamous cell carcinoma (SC/ ASC) and adenocarcinoma (AC) of the gallbladder. This analysis was conducted to include each HR reported by Zhang et al. (8).

There was high heterogeneity between the included studies, with an $\mathrm{I}^{2}$ value of $75.6 \%(\mathrm{P}=0.001)$ (Figure $\left.2 A\right)$. Thus, a random effects model was applied in this analysis. The pooled HR with 95\% CI between CD90 expression and OS was 2.56 (95\% CI: 1.42-4.62, $\mathrm{P}=0.002$ ), implying that the overexpression of CD90 was significantly associated with a poor OS in cancer patients (Figure $2 A$ ). When grouped according to cancer type, the subgroup with hepatobiliary cancers showed significant results (HR: 2.96, 95\% CI: 1.66-5.28, $\mathrm{P}<0.001$ ) (Figure $2 B$ ). 


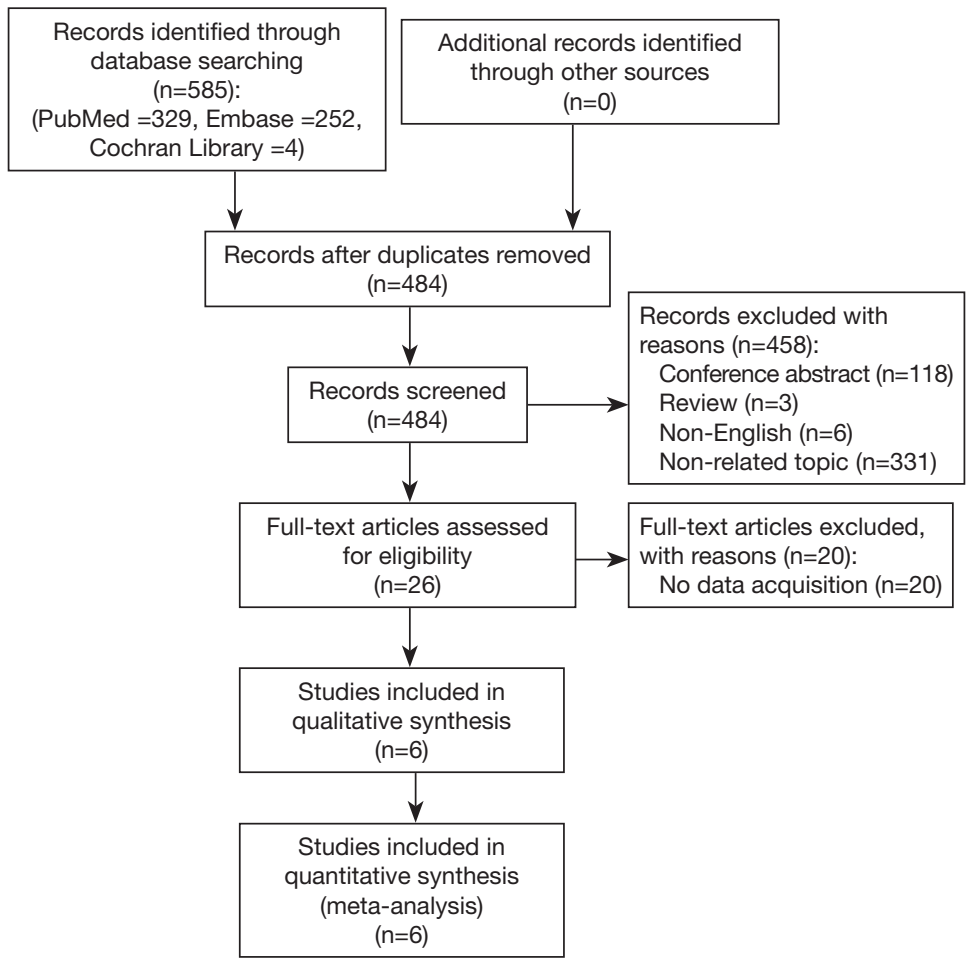

Figure 1 Flow diagram for the selection of studies to be included in the meta-analysis.

\section{The association between CD90 expression and disease-free survival (DFS)}

Of the included studies, four reported the relationship between CD90 expression and metastasis-free or recurrence-free or DFS. Here, metastasis-free or recurrence-free survival was considered and analyzed as DFS. There was high heterogeneity between the included studies, with an $\mathrm{I}^{2}$ value of $73.1 \%(\mathrm{P}=0.011)$ (Figure $\left.3 A\right)$. Thus, a random effects model was used. The pooled HR with 95\% CI between CD90 expression and DFS was 1.88 (95\% CI: $1.08-3.27, \mathrm{P}=0.025$ ), suggesting that the overexpression of CD90 is related to poor DFS in cancer patients (Figure $3 A$ ). In the subgroup analysis, hepatobiliary cancer revealed significant results (HR: 2.45, 95\% CI: $1.25-$ 4.78, $\mathrm{P}=0.009)$ (Figure 3B).

\section{The association between $C D 90$ expression and clinicopathological characteristics}

CD90 overexpression was significantly correlated with a larger tumor size (OR: 1.97, 95\% CI: 1.01-3.85, $\mathrm{P}=0.048$ ), higher tumor grade (OR: 2.72, 95\% CI: 1.33-5.54,
$\mathrm{P}=0.006)$, lymph node metastasis (OR: 3.66, 95\% CI: 1.14$11.78, \mathrm{P}=0.029)$, and higher tumor-node-metastasis (TNM) stage (OR: 4.79, 95\% CI: 2.28-10.04, $\mathrm{P}<0.001$ ) but not with age and sex (Table 2, Figure 4).

\section{Publication bias}

In the funnel plots, the distribution of the included studies was skewed to one side (Figure 5A,B). The Egger's test confirmed that there was publication bias (for OS, $\mathrm{P}=0.001$; for DFS, $\mathrm{P}=0.021$ ). Thus, we constructed filled funnel plots. The pooled HRs were still statistically significant (for OS, $\mathrm{P}=0.004$; for DFS, $\mathrm{P}=0.013$ ) (Figure $5 C, D$ ).

\section{Sensitivity analysis}

The sensitivity analysis revealed that the study published by Lobba et al. (4) had a significant impact on the initial pooled results (for OS, HR: 2.75, 95\% CI: 1.86-4.07; for DFS, HR: 2.16, 95\% CI: 1.43-3.26) (Figure 6). However, the pooled results were still statistically significant, implying that even after excluding the effects of individual studies, our initial results were meaningful. 


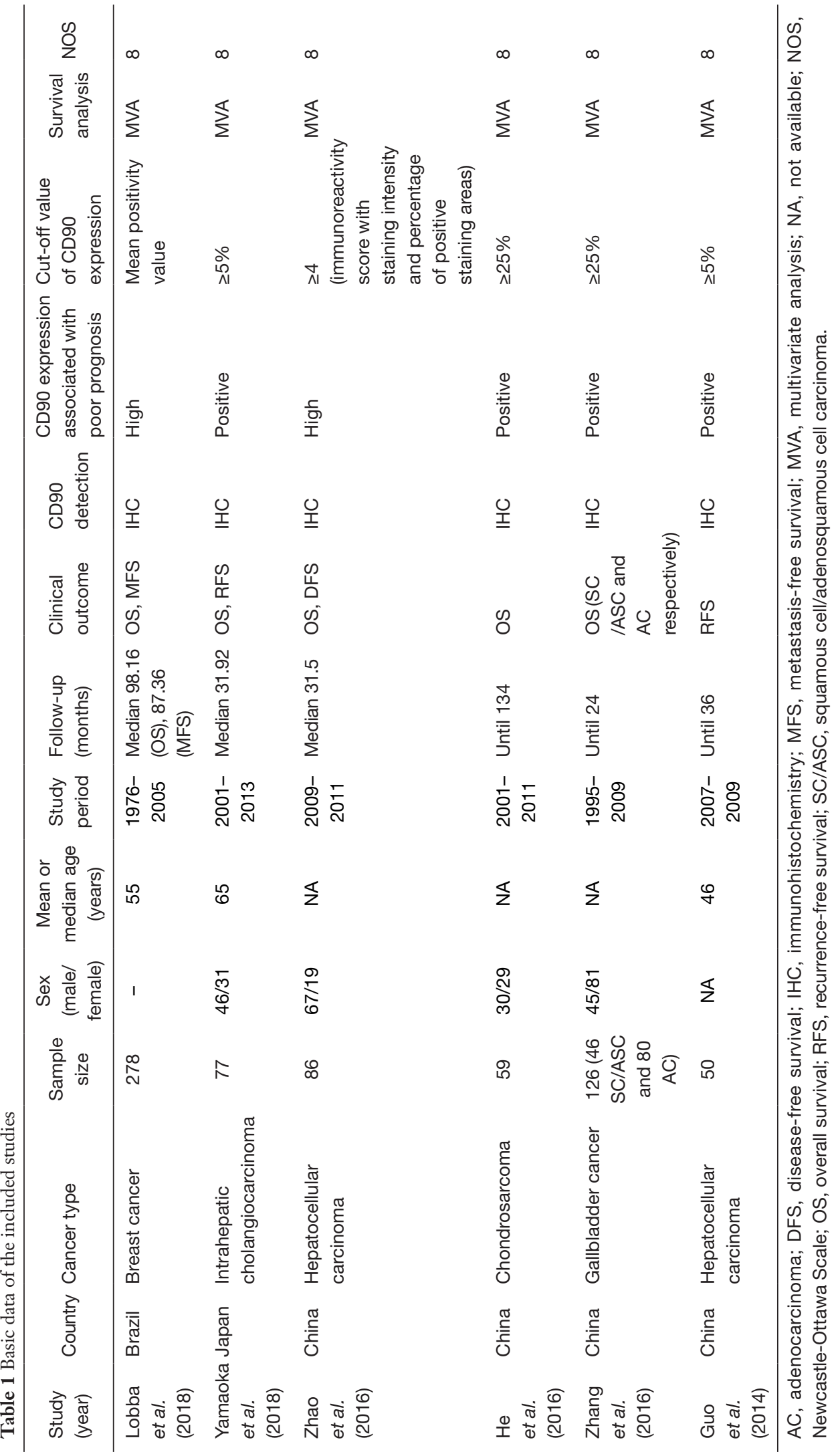



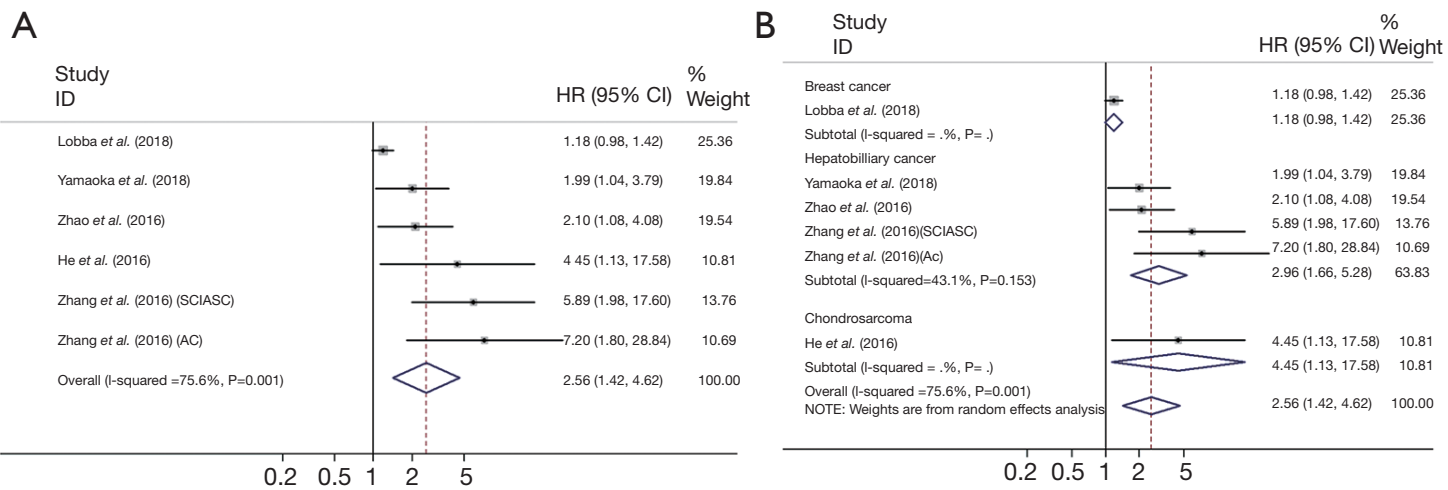

Figure 2 Forest plot of the association between CD90 expression and OS (A), stratified by cancer type (B).
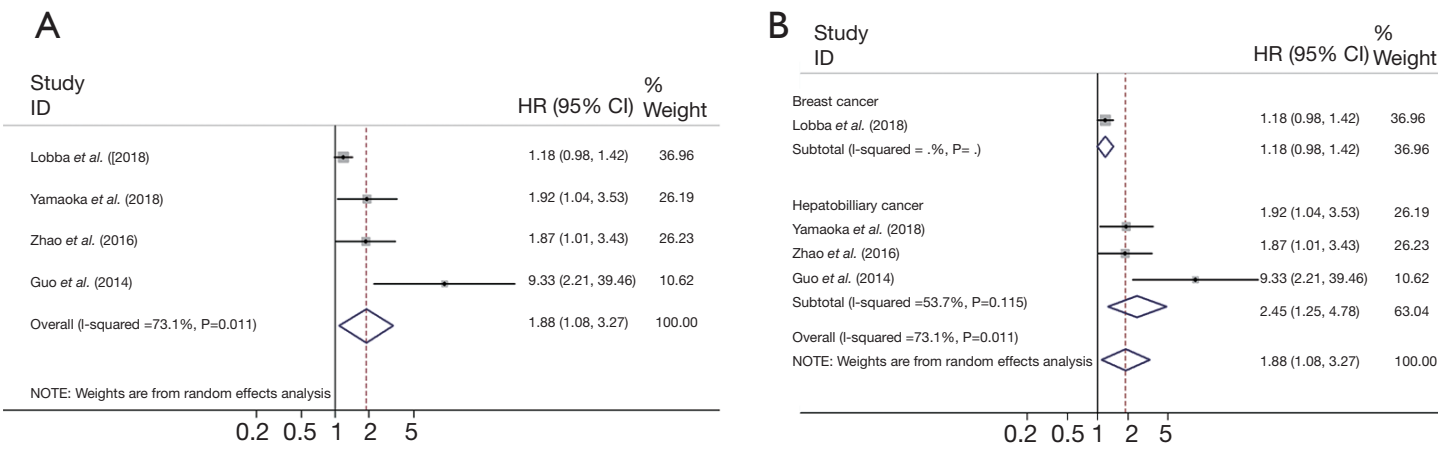

Figure 3 Forest plot of the association between CD90 expression and DFS (A), stratified by cancer type (B).

Table 2 The association between CD90 expression and clinicopathological characteristics in cancer patients

\begin{tabular}{|c|c|c|c|c|c|c|c|}
\hline \multirow{2}{*}{ Characteristic } & \multirow{2}{*}{$\begin{array}{l}\text { Number of } \\
\text { studies }\end{array}$} & \multirow{2}{*}{$\begin{array}{l}\text { Number } \\
\text { of patients }\end{array}$} & \multirow{2}{*}{$\begin{array}{l}\text { Pooled OR } \\
(95 \% \mathrm{Cl})\end{array}$} & \multirow{2}{*}{$P$ value } & \multicolumn{3}{|c|}{ Heterogeneity } \\
\hline & & & & & $\mathrm{I}^{2}(\%)$ & $P$ value & Model \\
\hline Age (old vs. young) & 3 & 423 & $\begin{array}{c}0.68 \\
(0.44-1.05)\end{array}$ & 0.078 & 0.0 & 0.552 & Fixed \\
\hline Sex (male vs. female) & 3 & 222 & $\begin{array}{c}0.74 \\
(0.41-1.33)\end{array}$ & 0.315 & 0.0 & 0.466 & Fixed \\
\hline Tumor grade (high vs. low) & 6 & 676 & $\begin{array}{c}2.72 \\
(1.33-5.54)\end{array}$ & 0.006 & 62.7 & 0.013 & Random \\
\hline $\begin{array}{l}\text { Lymph node metastasis } \\
\text { (present vs. absent) }\end{array}$ & 3 & 481 & $\begin{array}{c}3.66 \\
(1.14-11.78)\end{array}$ & 0.029 & 84.1 & $<0.001$ & Random \\
\hline
\end{tabular}

$\mathrm{Cl}$, confidence interval; OR, odds ratio; TNM, tumor-node-metastasis. 

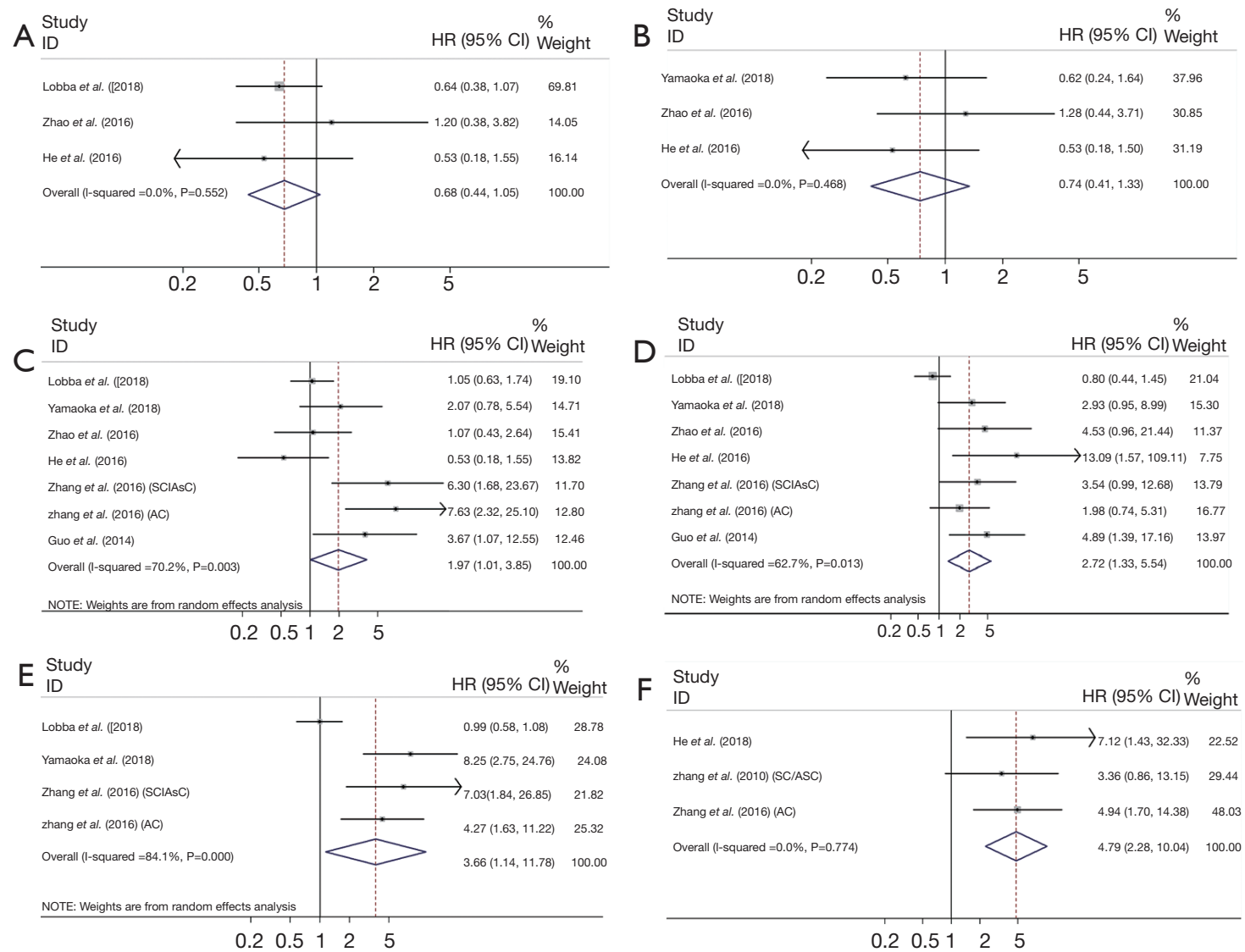

Figure 4 Forest plot of the association between CD90 expression and clinicopathological characteristics: (A) age, (B) sex, (C) tumor size, (D) tumor grade, (E) lymph node metastasis, and (F) tumor-node-metastasis stage.

\section{Discussion}

CD90 is a cell surface glycoprotein located on chromosome 11q22.3 (10). Although the function of CD90 has not been fully identified, it is involved in intercellular and cell-tomatrix interactions, apoptosis, cell adhesion and migration, and neurite overgrowth modulation (10).

In various cancers, CD90 expression has been associated with cancer stem cells, which induce cancer initiation and metastasis (2). For example, several researchers have reported that CD90 expression is increased in hepatic cancer tissue compared with normal or cirrhotic liver tissue and is higher in poorly differentiated than in welldifferentiated cancer (2). In hepatocellular carcinoma, CD90 expression facilitates the migration of cancer cells by causing the upregulation of EpCAM and downregulation of E-cadherin (2). In melanoma, CD90 is expressed in endothelial cells, promoting the metastasis of melanoma cells with integrin (2). CD90 is also expressed in the tumor front by invading breast cancer cells (2).

In addition, recent studies have shown that CD90 expression is related to the prognosis of cancer patients. Yamaoka et al. (7) reported that CD90 expression was significantly associated with lymph node metastasis and could be an independent prognostic factor in intrahepatic cholangiocarcinoma. Zhang et al. (8) revealed that CD90 overexpression was correlated with a poor differentiation, large tumor size, lymph node metastasis, and substantial invasiveness in SC/ASC and AC of the gallbladder and that patients with positive CD90 expression had shorter OS than those with negative CD90 expression. Moreover, Zhao et al. (9) demonstrated that CD90 expression was correlated with the pathologic grade, stellate lesion, portal vein tumor thrombi, and recurrence and that high CD90 expression could predict unfavorable prognosis in hepatocellular carcinoma. Guo et al. (5) also showed CD90 expression to be significantly associated with early recurrence in hepatocellular carcinoma. He et al. (6) identified that 

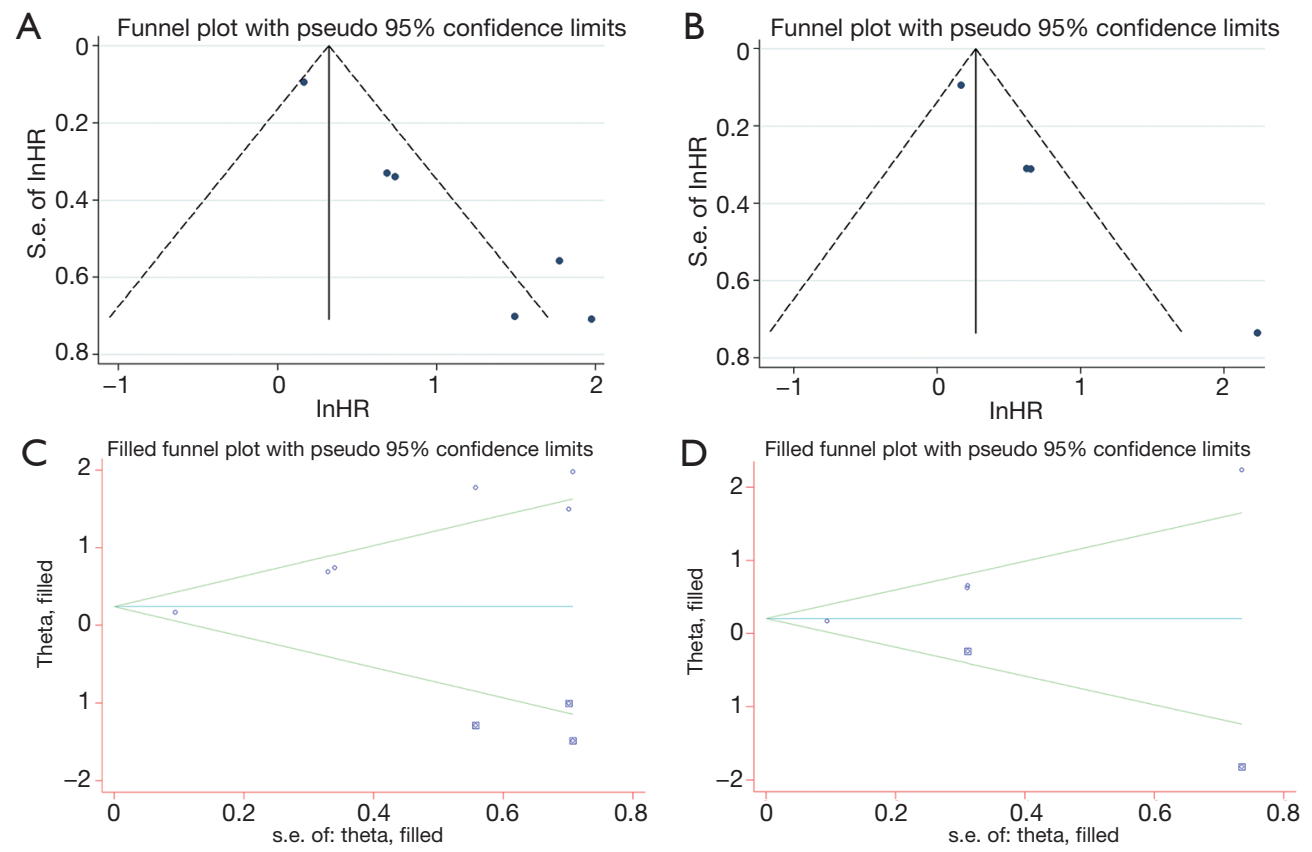

Figure 5 Funnel plot and filled plot of the association between CD90 expression and OS (A,C) and DFS (B,D).

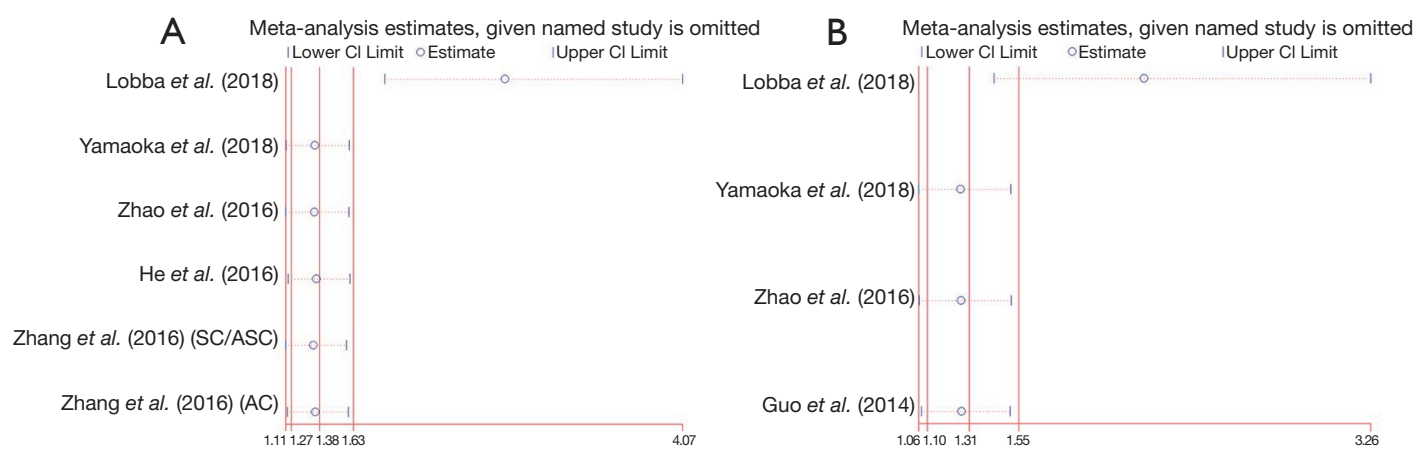

Figure 6 Sensitivity analysis of the association between CD90 expression and OS (A) and DFS (B).

CD90 expression was negatively correlated with OS in chondrosarcoma, and Lobba et al. (4) reported the relationship between CD90 expression and the prognosis of breast cancer patients.

In the present study, we performed a meta-analysis for OS and DFS and demonstrated that CD90 overexpression was associated with a poor prognosis in cancer patients. We also found that CD90 overexpression was significantly correlated with a larger tumor size, higher tumor grade, lymph node metastasis, and higher TNM stage.

There are some limitations to the present study. First, the number of studies included was small, and all of them were published in Asia, with the exception of one study. Moreover, the heterogeneity among the included studies was significant probably because several baseline characteristics varied, such as cancer type, sample size, and criteria for determining CD90 expression. However, the source of the heterogeneity was not revealed because of the limited number of studies. Second, the criteria for determining CD90 expression differed from study to study. Four studies evaluated CD90 expression by positive fraction, two of which were based on $25 \%$, and the other two were based on $5 \%$. Among the other two studies, CD90 expression was determined by combining the staining intensity and the percentage of positive staining areas, whereas the other applied CD90 expression based on the 
mean positive value. Finally, there have been recent reports that the expression and function of CD90 are dependent on the cellular context and the tumor microenvironment, so our analysis may be limited. We hope that further research on the relationship between CD90 expression and the prognosis of cancer will be conducted and that better metaanalyses could be conducted.

In summary, we systematically analyzed the prognostic and clinicopathological value of CD90 expression in patients with cancer. CD90 overexpression could predict poor prognosis in patients with cancer and may hence be a potential prognostic biomarker.

\section{Acknowledgments}

Funding: This work was supported by the Soonchunhyang University Research Fund.

\section{Footnote}

Reporting Checklist: The authors have completed the PRISMA reporting checklist. Available at https://dx.doi. org/10.21037/tcr-21-266

Conflicts of Interest: All authors have completed the ICMJE uniform disclosure form (available at https://dx.doi. org/10.21037/tcr-21-266). The authors have no conflicts of interest to declare.

Ethical Statement: The authors are accountable for all aspects of the work in ensuring that questions related to the accuracy or integrity of any part of the work are appropriately investigated and resolved.

Open Access Statement: This is an Open Access article distributed in accordance with the Creative Commons Attribution-NonCommercial-NoDerivs 4.0 International License (CC BY-NC-ND 4.0), which permits the noncommercial replication and distribution of the article with the strict proviso that no changes or edits are made and the original work is properly cited (including links to both the formal publication through the relevant DOI and the license). See: https://creativecommons.org/licenses/by-nc-nd/4.0/.

\section{References}

1. Karley D, Gupta D, Tiwari A. Biomarker for Cancer: A great Promise for Future. World J Oncol 2011;2:151-7.

2. Kumar A, Bhanja A, Bhattacharyya J, et al. Multiple roles of CD90 in cancer. Tumour Biol 2016;37:11611-22.

3. Rege TA, Hagood JS. Thy-1, a versatile modulator of signaling affecting cellular adhesion, proliferation, survival, and cytokine/growth factor responses. Biochim Biophys Acta 2006;1763:991-9.

4. Lobba ARM, Carreira ACO, Cerqueira OLD, et al. High CD90 (THY-1) expression positively correlates with cell transformation and worse prognosis in basal-like breast cancer tumors. PLoS One 2018;13:e199254.

5. Guo Z, Li LQ, Jiang JH, et al. Cancer stem cell markers correlate with early recurrence and survival in hepatocellular carcinoma. World J Gastroenterol 2014;20:2098-106.

6. He L, Yang Z, Li Z. The clinical pathological significance of Thy 1 and CD49f expression in chondrosarcomas. Pathol Res Pract 2016;212:636-42.

7. Yamaoka R, Ishii T, Kawai T, et al. CD90 expression in human intrahepatic cholangiocarcinoma is associated with lymph node metastasis and poor prognosis. J Surg Oncol 2018;118:664-74.

8. Zhang DH, Yang ZL, Zhou EX, et al. Overexpression of Thy1 and ITGA6 is associated with invasion, metastasis and poor prognosis in human gallbladder carcinoma. Oncol Lett 2016;12:5136-44.

9. Zhao RC, Zhou J, Chen KF, et al. The prognostic value of combination of CD90 and OCT4 for hepatocellular carcinoma after curative resection. Neoplasma 2016;63:288-98.

10. Lu JW, Chang JG, Yeh KT, et al. Overexpression of Thy1/ CD90 in human hepatocellular carcinoma is associated with $\mathrm{HBV}$ infection and poor prognosis. Acta Histochem 2011;113:833-8.
Cite this article as: Koh HM, Lee HJ, Kim DC. Prognostic and clinicopathological value of CD90 expression in cancer patients: a systematic review and meta-analysis. Transl Cancer Res 2021;10(7):3356-3363. doi: 10.21037/tcr-21-266 